\title{
Statistics of large impulsive magnetic events in the auroral zone
}

\author{
Kyle Reiter $^{1, *}$ (D), Sébastien Guillon ${ }^{2}$ (D), Martin Connors ${ }^{1,3}$ (D), and Brian Jackel ${ }^{1}$ \\ ${ }^{1}$ Department of Physics and Astronomy, University of Calgary, Calgary T2N 1N4, AB, Canada \\ 2 TransÉnergie-Équipement, Hydro-Québec, Montréal H5B 1H7, QC, Canada \\ ${ }^{3}$ Athabasca University Observatories, Athabasca T9S 3A3, AB, Canada
}

Received 11 February 2021 / Accepted 11 July 2021

\begin{abstract}
Impulsive geomagnetic variations, latitudinally localized to the auroral zone, have been observed from 2015 to 2020 . These impulsive events have been observed mostly in the pre-midnight sector as upward vertical perturbations. Diurnal variations in geomagnetically-triggered harmonic distortion events observed in Hydro-Québec's Système de Mesure de Décalage Angulaire (SMDA) synchrophasor measurement system found to have a peak in the number of events around midnight. This was similar to diurnal rates of negative $B_{z}$ geomagnetic impulsive events observed at nearby auroral zone magnetometers. Superposed epoch analysis demonstrates the impulses are regularly associated with increases in harmonic distortion observed at a nearby substation transformer. These large impulsive vertical geomagnetic perturbations appear to be local vortical ionospheric disturbances.
\end{abstract}

Keywords: impulsive geomagnetic events / geomagnetically induced currents / auroral zone ionosphere

\section{Introduction}

The nightside auroral zone ionosphere has long been a subject of study (Fukushima, 1994), being a region of dynamic current structures which interconnect with the inner magnetosphere (McPherron et al., 1973). During geomagnetically active periods, changing ionospheric currents occur in the auroral zone. The resulting geoelectric fields present a potential hazard: the excitation of geomagnetically induced currents (GIC) in conducting infrastructure such as power transmission networks and pipelines (Bolduc, 2002; Boteler, 2003; Lundstedt, 2006). The relatively low frequency of GIC variations, compared to the operating frequency of the power transmission network, means GIC can produce a quasi-DC voltage bias of transformers. This can saturate the core of the transformer, producing a harmonic distortion of the transformer voltage (Boteler, 2001). For auroral zone power infrastructure, substorms, pulsations, and horizontal geomagnetic perturbations constitute major drivers of geomagnetic activity, which may cause power system disruption (Pulkkinen et al., 2003; Kappenman, 2005; Stauning, 2013; Ngwira et al., 2015; Guillon et al., 2016; Boteler, 2019). Large impulsive magnetic events may act as another source of GIC. If these events were to have a prominent vertical component, Faraday's Law would indicate that the resultant large vertical magnetic flux changes produced from these events should induce similarly large horizontal geoelectric fields. Commonly,

\footnotetext{
*Corresponding author: kyle.reiterl@ucalgary.ca
}

when modeling geoelectric fields based on ground magnetometer measurements, $B_{z}$ measurements are neglected, using the plane-wave assumption (Wait, 1982; Boteler \& Pirjola, 2017). We present a statistical study including the occurrence of $B_{z}$ impulsive events and their relation to GIC.

Harmonic distortion (HD) is often used as an indicator of the stress applied to a power transmission network due to GIC (Bolduc, 2002; Clilverd et al., 2020; Rodger et al., 2020). GIC in transformers within a power transmission network has a spectral content with frequencies much lower than the normal $60 \mathrm{~Hz}$ operating frequency of the network. Therefore they can be approximated as quasi-DC currents. Transformer cores within a network may become partially saturated due to the introduction of GIC, resulting in distortion of the AC signal. This can be quantified as HD (Albertson et al., 1981). The Hydro-Québec network has been extensively instrumented to measure HD, which provides a continuous record of space weather impacts to the network (Kamwa et al., 2006). As a result, this study uses $\mathrm{HD}$ as an indicator of GIC-driven stress on the power transmission network.

Previous statistical studies of impulsive geomagnetic events examined impulses measured on the dayside in the cusp regions (Lanzerotti et al., 1986, 1991). One study considered auroralzone impulsive events but limited horizontal geomagnetic perturbations (Moretto et al., 2004). Some more recent studies have looked at impulsive geomagnetic perturbations on the nightside but have principally focused on impulsive perturbations in the polar cap (Engebretson et al., 2019a) or in a storm context 
(Engebretson et al., 2019b). This study seeks to characterize the latitudinal distribution of impulsive magnetic events, their diurnal distribution in magnetic local time (MLT), their amplitude, which component is dominant, and their relation to power network disturbances. We characterize these events in the aggregate and find that most geoeffective events are associated with vertical disturbances.

\section{Materials and methods}

\subsection{Source data}

We analyze data from two sources: ground-based magnetometers and synchrophasor measurements of voltage distortion at substation transformers. The region of interest for the study is shown in Figure 1. The fluxgate magnetometers used in this study were of the same design used by time history of events and macroscale interactions during substorms (THEMIS) ground-based observatories (GBO; Harris et al., 2009). Magnetic field data is from selected stations in the Athabasca University THEMIS UCLA Magnetometer Network eXpansion (AUTUMNX; Connors et al., 2016) ranging from subauroral to polar cap regions. AUTUMNX stations in this study were Radisson (RADI, $63.25^{\circ}$ geomagnetic latitude), Kuujjuarapik (KJPK, 64.73 ${ }^{\circ}$ ), Inukjuak (INUK, 67.92 ${ }^{\circ}$ ), and Salluit (SALU, $71.71^{\circ}$ ). Corrected Geomagnetic latitudes for these stations were determined by Connors et al. (2016). The magnetometers are located along a longitudinal chain at an MLT where midnight is at 5 UT. Magnetometer data was collected and stored at a $2 \mathrm{~Hz}$ cadence, in north $(X)$ west to east $(Y)$, and positive downward vertical $(Z)$ components. Data from the AUTUMNX network has been analyzed from January 1, 2015, through June 30, 2020.

Hydro-Québec total harmonic distortion data from June 2014 through May 2017 was collected on a 4 s cadence from automatically selected periods of elevated disturbance identified by Hydro-Québec. This is measured using Hydro-Québec's Système de Mesure de Décalage Angulaire (SMDA) synchrophasor measurement system (Kamwa et al., 2006; Guillon et al., 2016). Total harmonic distortion is a measure of the voltage distortion at the substation transformer. These are archived when even-harmonic distortion (EHD) of voltages at designated substation transformers throughout the Hydro-Québec network exceeds substation-specific thresholds. This triggers the recording of high-resolution HD data for approximately $30 \mathrm{~min}$. The start time of SMDA data collection serves as the event time used. EHD was collected at a $1 \mathrm{~Hz}$ cadence, and is defined by Hydro-Québec as:

$$
\mathrm{EHD}=\frac{\sqrt{V_{2}^{2}+V_{4}^{2}+V_{6}^{2}+V_{8}^{2}}}{\sqrt{V_{f}^{2}+V_{2}^{2}+V_{4}^{2}+V_{6}^{2}+V_{8}^{2}}} .
$$

$V_{n}$ corresponds to the amplitude of the $n$th harmonic of voltage at the fundamental $60 \mathrm{~Hz}$ operating frequency, $V_{f}$. Odd harmonic distortion information was not used in the analysis due to a lack of availability. While the high-resolution HD data is available only on a limited and activity-triggered basis, lower resolution EHD data is available on a continuous basis. The EHD data is a continuous time series from 2015 to 2019 ,

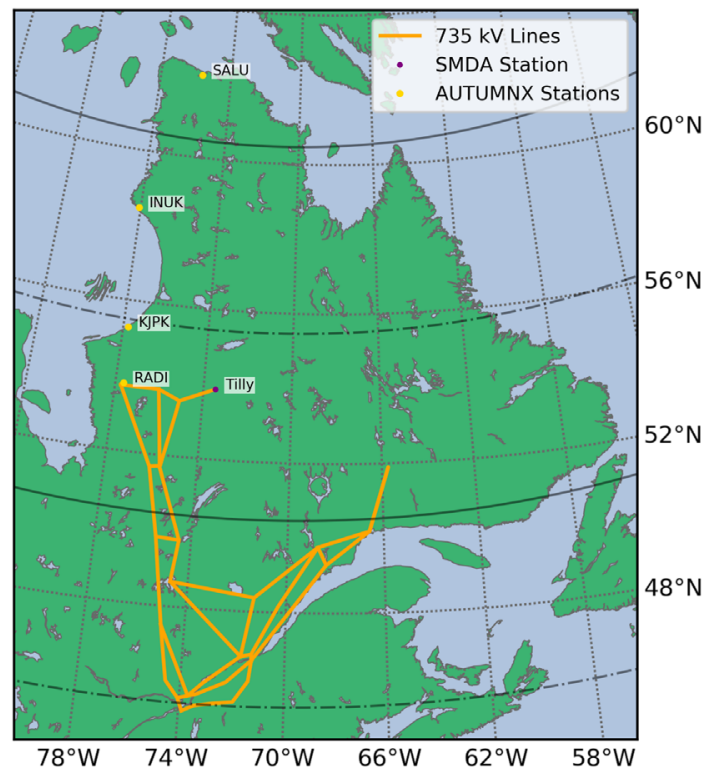

Fig. 1. The region of interest. Magnetometers used are indicated in yellow. $735 \mathrm{kV}$ Hydro-Québec transmission lines are shown in orange, with the Tilly transformer substation from which EHD data is used indicated in purple. Dot-dash lines indicate $55^{\circ}$ and $65^{\circ}$ corrected geomagnetic latitude, solid lines indicate $60^{\circ}$ and $70^{\circ}$ latitude.

collected from the Tilly substation (about $5^{\circ}$ south of Inukjuak) as seen in Figure 1.

\subsection{Geomagnetic extremum identification}

We designed an algorithm to select for geomagnetic perturbations which were short in duration (minutes), large in amplitude (100s of nT), and non-periodic. This led to a set of criteria similar to those used by Lanzerotti et al. (1991), as detailed in Figure 2. While large impulsive magnetic events are associated with large $\mathrm{d} B / \mathrm{d} t$, these are distinguished from monotonic decreases or increases in the field by an extremum. $\mathrm{d} B / \mathrm{d} t$ calculated from field measurements at the extremum will be small in magnitude, reflecting the flat-topped characteristic of impulsive events. As an initial selection criterion to identify local extrema, a low $\mathrm{d} B / \mathrm{d} t$ threshold was used. Subsequent removal of all but the largest and smallest values from 5-min windows in the data set was used to identify the largest magnitude impulsive events in the window. A $200 \mathrm{nT}$ offset of the median values of 4-min windows on both sides of the extrema was chosen. This selects for large perturbations, which may result in the induction of significant GIC. Impulsive events are sorted into positive or negative events by their relation to the median component values in the 4 min preceding and following the extremum. Examples of events that do and do not meet the selection criteria can be found in Annex A.

The power spectral density was determined from a 2-h window (14,400 points) around the event, which is meansubtracted with a Hanning (weighted cosine) window applied (Kanasewich, 1974):

$$
w(n)=0.5-0.5 \cos \left(\frac{2 \pi n}{M-1}\right) \quad 0 \leq n \leq M-1 .
$$




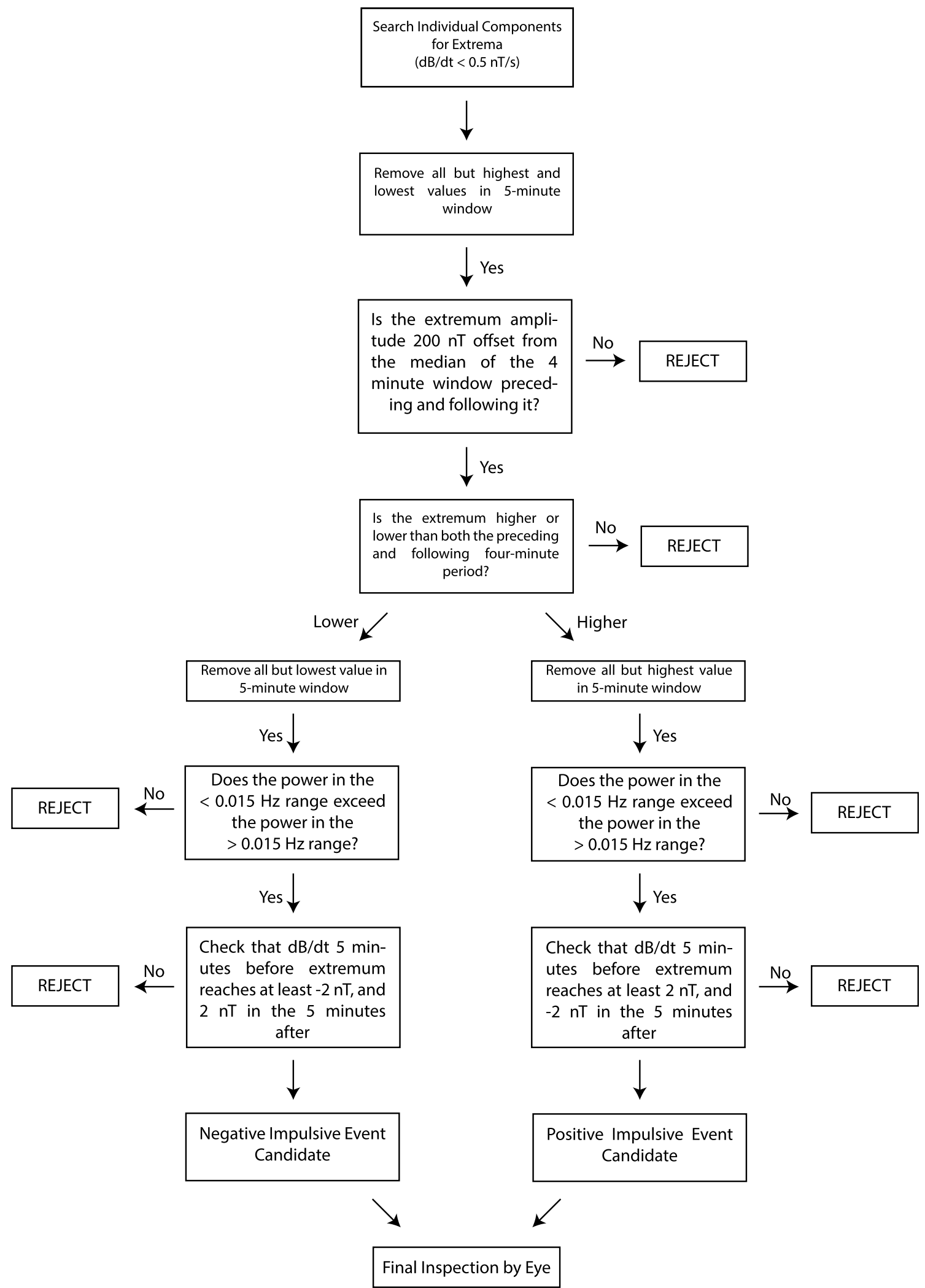

Fig. 2. A flow diagram depicting the selection criteria for impulsive events. Selection is conducted on a component-by-component basis for each individual magnetometer. 



Fig. 3. Left (a)-(c): Diurnal variations in impulsive magnetic events per hour found from 2015 to 2020 in Inukjuak, Quebec. Right (d)-(f): Distribution of magnitude of 8-min component ranges around identified impulsive events per 100 nT bin. Vertical scales vary between components to better convey diurnal trends. Total event numbers are indicated in bold gray for each component. Blue shading indicates negative impulses, red shading positive.

$w(n)$ defines the windowing function in which the time-series data is multiplied element-wise by, $n$ is the index number, and $M$ is the size of the window. Events with missing data were not used in the analysis. The power spectrum was calculated using a discrete Fourier transform implemented using a fast Fourier transform algorithm for each event.

The power content in the $<1.5 \mathrm{mHz}$ range was compared to the $1.5-16.7 \mathrm{mHz}$ range, and events, where more power was contained in the higher frequency range, were discarded. This principally removes pulsation events with periodicities similar to the 8-min selection window, as well as candidate events which are the result of instrumentation issues. The last step in computer-identification of events was to check that the $\mathrm{d} B / \mathrm{d} t$ in the 10 -min window immediately preceding and following the candidate event has the correct magnitude and sign for an impulsive event of its identified polarity. This removes events that may have local component extrema but are not characterized by sudden changes. Finally, candidates are examined by eye to remove any events characteristic of instrumentation faults. This step also removes any events or variations which are insignificant when compared with proximate geomagnetic activity. This step results in a relatively small number of candidate events being rejected: in the case of the Inukjuak magnetometer, only 69 of 996 total events. Similar human interventions have been deemed necessary in previous impulsive event statistical studies (Lanzerotti et al., 1991).

While the selection algorithm takes inspiration from Lanzerotti et al. (1991), the selection criteria have been modified to select for similar but distinct types of magnetic perturbations. Principal differences include the larger and more selective magnetic component deflection threshold and the use of spectral power content as a filter. Most notably, our analysis allows for events occurring in a single geomagnetic field component. This broadens our analysis compared to that of Lanzerotti et al. (1991), allowing for a comparison of the relative prevalence of horizontal and vertical impulsive events.

\section{Analysis}

\subsection{Diurnal variations}

Patterns in the occurrence of impulsive events were identified by constructing histograms using event magnitude and MLT hourly bins. Diurnal variations and the magnitude distribution of magnetic impulsive events for the field components 

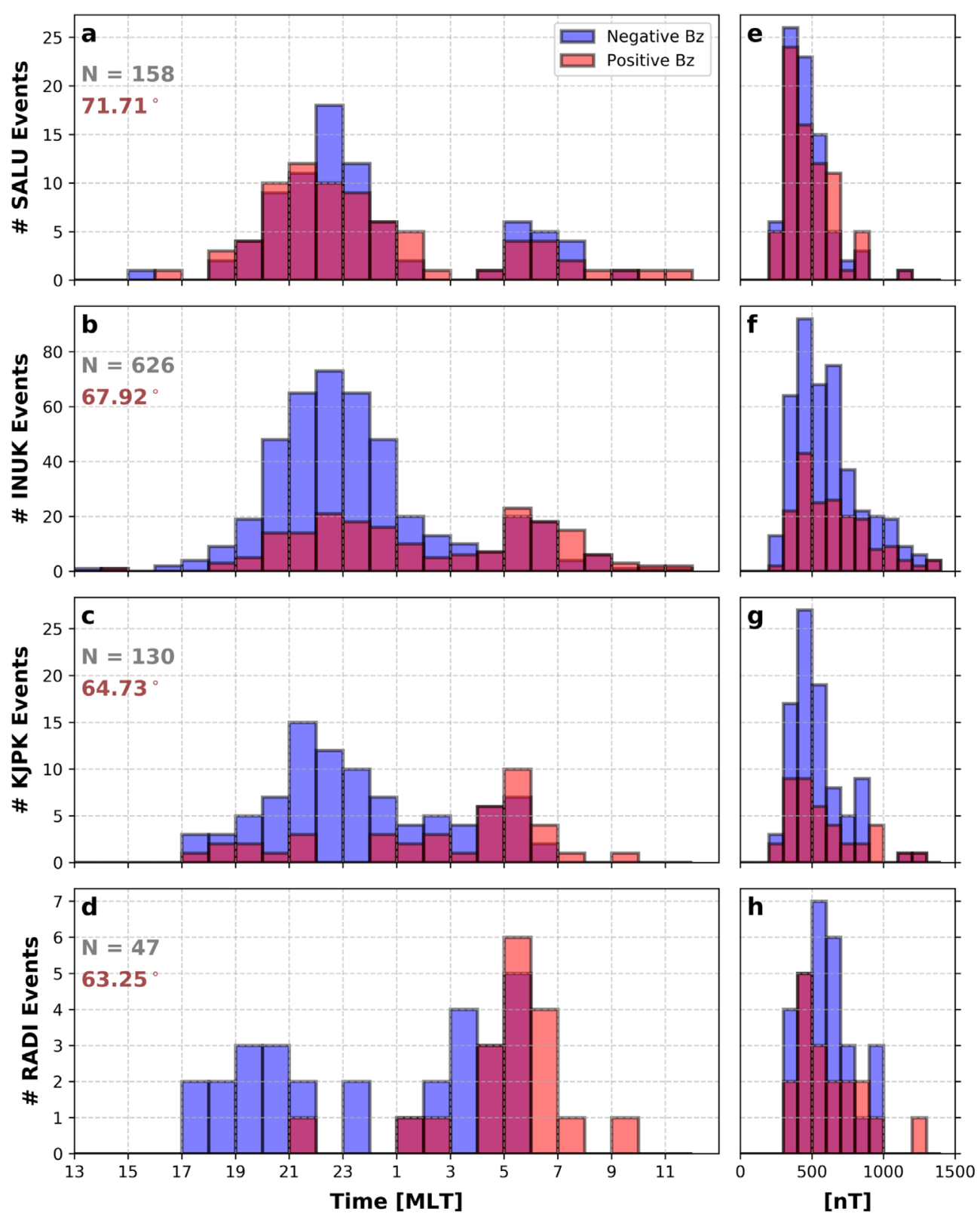

Fig. 4. Left (a)-(d): Magnetic local time distribution of impulsive events per hour as recorded at selected AUTUMNX locations. Right (e)-(h): Distribution of magnitude of 8-min $B_{z}$ ranges around identified impulsive events per $100 \mathrm{nT}$ bin. Vertical scales vary between magnetometers to better convey diurnal trends. Total event numbers are indicated in bold gray for each magnetometer, with corrected geomagnetic latitude indicated in dark red indicated underneath. Blue shading indicates negative impulses, red shading positive.

at Inukjuak, Québec are seen in Figure 3. This auroral-zone magnetometer shows a distinct peak in the occurrence of impulses in all components in the pre-midnight and morning sectors. The rate of occurrence and magnitude of $B_{z}$ perturbations are significantly higher than those of other components. The diurnal distribution of impulsive events for magnetometer sites from sub-auroral through polar-cap latitudes can be seen in Figure 4. The total number of $B_{z}$ events for each magnetometer can be seen in gray in panels a-d. High rates of occurrence of impulsive $B_{z}$ perturbations are narrowly latitudinally localized. As seen in panel $\mathrm{f}$ of Figure 4, an extended tail of large magnitude impulsive events is evident at Inukjuak, which also experienced by far the most $B_{z}$ impulsive events. The magnitude of these impulses is significantly larger than those in similar previous studies (Lanzerotti et al., 1991; Moretto et al., 2004). The largest impulsive events see an 8-min range in excess of 1000 nT in the vertical component. The 8-min range is defined analogously to the hourly range of one geomagnetic component, for one magnetometer (Hruska \& Coles, 1987; Danskin \& Lotz, 2015). Perturbations in multiple components were seen for many events, though usually not of a magnitude to qualify as simultaneous impulsive events in multiple components. For example, only 38 of 436 negative $B_{z}$ impulsive events at Inukjuak had coincident impulsive events in another component.

It is of interest to power network operators to know at what local times heightened levels of HD are prevalent in their 


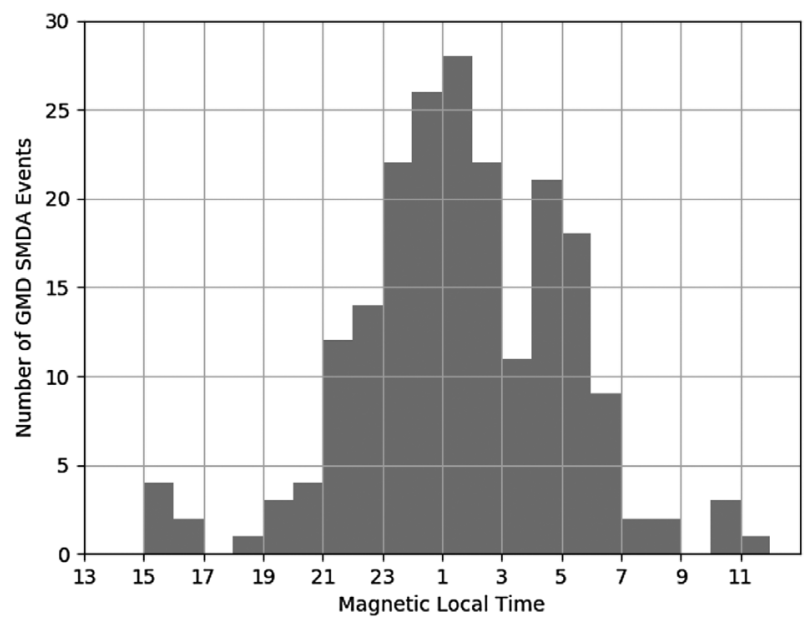

Fig. 5. Local time distribution of harmonic distortion events observed by Hydro-Québec SMDA monitoring system, 20142017. Local time was taken from an average of longitudinal extremes of substations which may trigger the event.

networks. The diurnal distribution of heightened HD within a power transmission network may also provide insight into potential drivers of HD and related GIC. Figure 5 shows the local-time distribution of the EHD threshold triggered HD events in the Hydro-Québec network, which occur predominantly on the nightside. Peaks are seen around midnight and in the morning sector around 5 MLT. Substorm activity has been previously found to predominantly occur near midnight (Frey et al., 2004). The diurnal pattern of heightened HD activity is consistent with both substorm-driven and impulse-driven GIC. It is expected that the diurnal distribution of HD will be somewhat broader in MLT than that in magnetic data from the AUTUMNX meridian alone due to the longitudinal extent of the power network substations whose HD levels were monitored. We now directly compare HD levels occurring around these impulsive events with periods of similar global electrojet activity, as indicated by electrojet indices.

\subsection{Superposed epoch analysis}

We now look at the geoeffectiveness of the impulsive vertical events. Some events in Figure 4 appear to be substorm associated, and we would like to minimize the number of this type of event in our comparative analysis. Intensification of the westward auroral electrojet will result in a positive vertical magnetic component perturbation detectable to the north, and a negative perturbation to the south (Kisabeth \& Rostoker, 1974). For an intensified westward electrojet, one would expect a downward perturbation to the vertical component to the north and upward (negative $B_{z}$ ) to the south. This tendency can be seen in the increased number of positive $B_{z}$ perturbations in the pre-midnight sector at Salluit, and negative $B_{z}$ perturbations in the pre-midnight sector at Kuujjuarapik. Inukjuak's auroral latitude means that it is more often closer to the latitude of the electrojet. As a result, it should experience less frequent latitudinally offset electrojet enhancements which would produce $B_{z}$ perturbations than sites to its north or south. Without introducing additional selection criteria, using Inukjuak geomagnetic field data will minimize the number of substorm-related events we use in our analysis. Further, to select unusually large impulses, we have taken the 50 largest negative $B_{z}$ impulsive events at Inukjuak.

Superposed epoch analysis for 2-h windows around the negative $B_{z}$ events selected can be seen in Figure 6. Median component values in bold black in panels a-c for separate geomagnetic components demonstrate some average variation in the horizontal geomagnetic field $(a, b)$, but vertical perturbations (c) are much larger. Considerable individual event variability in all components can also be seen.

Averaged EHD data from the Tilly substation are found in panels e and k of Figure 6. This EHD data is taken from the continuous EHD record rather than the event-limited HD data used for Figure 5. Median EHD response (e) indicates increased geoeffectiveness of negative $B_{z}$ impulsive events, though individual events (d) do evidence variability in response. SuperMAG auroral indices SML and SMU (analogous to AL and AU; Newell \& Gjerloev, 2011; Gjerloev, 2012) indicate some strengthening of the westward electrojet prior to the impulsive events, corresponding with a slight median decrease in the SML index. Panels g-1 of Figure 6 shows data from randomly selected periods with SME values within $20 \mathrm{nT}$ of the SME level at the impulsive event for the same magnetometer and substation transformer. No median deviations in EHD response are seen for periods with equivalent SME in panel $\mathrm{k}$. This provides evidence for the locality of these vertical impulsive events. Electrojet indices reflect the development of substorm phenomena but do not well capture the magnitude of local geomagnetic variability associated with these impulsive events.

\section{Discussion}

We have presented evidence of regularly occurring auroralzone impulsive events. These are principally, though not solely, vertical in nature and almost entirely found on the nightside. They appear to be localized in latitudinal occurrence and are principally negative $B_{z}$ perturbations. The locality and latitudes of occurrence indicate that this is an ionospheric auroral electrojet-related phenomenon. The vertical nature of the disturbances is evidence of vortical or anti-parallel directed ionospheric currents, which are also short-lived in duration due to the impulsive nature of the $B_{z}$ perturbations. Lanzerotti et al. (1986) determined a possible vortical Hall current system driven by field-aligned currents (FAC) that would produce a vertical geomagnetic perturbation. The magnitude of the impulsive events in the present study are larger, with most impulsive $B_{z}$ events occurring at auroral zone latitudes, but the proposed ionospheric current system is consistent with our observations. Traveling convection vortices (TCV) have been previously observed in the ionosphere (Friis-Christensen et al., 1988; Engebretson et al., 2013). Evidence has also been found of TCVs, which are geoeffective (Belakhovsky et al., 2019; Chinkin et al., 2021). However, TCVs occur near the dayside with stronger periodicities than we selected. Some $B_{z}$ impulsive events seen in Figure 4, particularly those in Salluit and Radisson, are likely signatures of substorm electrojet enhancement (Kisabeth \& Rostoker, 1974) rather than ionospheric vorticity, though this is less true of the largest $B_{z}$ impulses seen at Inukjuak. 

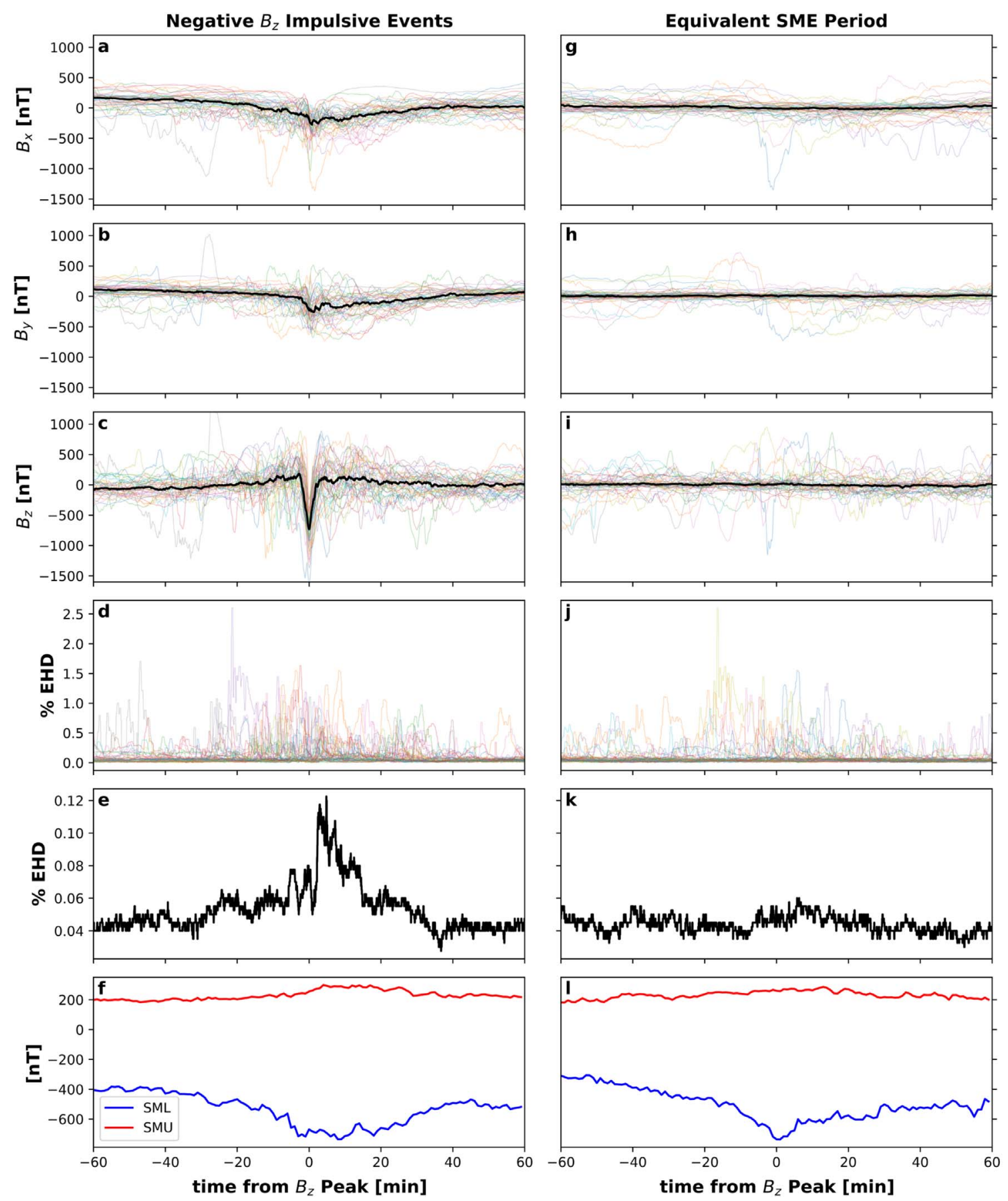

Fig. 6. Left (a)-(f): Superposed epoch analysis of magnetometer data from Inukjuak QC and EHD from the Tilly substation in the HydroQuébec network (median values in bold black). Superposed epoch trigger is the negative $B_{z}$ extremum for the 50 largest negative $B_{z}$ impulsive events. Median SMU (red) and SML (blue) for these 2-h windows are shown in the bottom panel. Individual EHD traces are colorized in d, median values shown in e. Right (g)-(l): Inukjuak QC magnetometer readings for equivalent SME periods at the $B_{z}$ extrema, and corresponding median EHD values at the Tilly substation. All SME values have been minimum EHD subtracted for each two-hour period. Events without HD data from Tilly were not used (5 of 50 events).

It may be surmised through both the auroral latitude and pre-midnight peak occurrence of these impulsive negative $B_{z}$ events that they could be associated with the Harang reversal (Kamide, 1978). This location, where equatorward of the eastward electrojet, the westward electrojet, can be found (Harang, 1946) provides a potential source of current vorticity. However, the largest $B_{z}$ impulses are both larger in amplitude and shorter in duration than previous ground-based magnetometer observations of the Harang reversal (Kamide \& Vickrey, 1983; Untiedt \& Baumjohann, 1993). An impulsive intensification of eastward and westward convection electrojets could provide a mechanism to intensify the transient $B_{z}$ signature associated with the Harang 
reversal. While reducing SML (and enhancement of the westward electrojet) is evident in panel $\mathrm{f}$ of Figure 6, the enhancement of the eastward electrojet, as indicated by an increase in SMU, is relatively small. Counter-clockwise vortices have also been previously associated with strengthening Region 2 upward FAC in the proximity of the Harang reversal, through combined Poker Flat Incoherent Scatter Radar (PFISR) and THEMIS GBO all-sky imager (ASI) observations of substorm events (Zou et al., 2009).

Unsurprisingly, that GIC-driven HD events in high latitude sub-auroral power transmission infrastructure would predominantly occur on the nightside around midnight, proximate to the peak of substorm occurrence. However, we have additionally found evidence of median increases in nearby substation transformer HD levels during vertical impulsive events, even when compared with periods with similar SME. The geographic distance between the substation and magnetometer suggests that these impulsive events are localized phenomena and that their resultant geoelectric fields may be distributed on a scale of 100 s of kilometers.

These impulsive events have ground magnetic signatures that are predominantly vertical in nature, regularly occur, exhibit similar diurnal variations to those of geomagnetically-driven HD events, and are found to excite larger median harmonic distortion responses from nearby substation transformers than periods with equivalent electrojet indices. This indicates that these are events of operational interest to power transmission infrastructure operators at auroral and high-latitude sub-auroral latitudes. Additionally, geoelectric field models which neglect vertical geomagnetic perturbations may systematically underrepresent the magnitude of GIC which such impulsive events may produce.

\section{Conclusions}

We present evidence that there are regularly occurring vertical impulsive events in the geomagnetic field at auroral zone latitudes. These events predominantly occur on the nightside in the pre-midnight sector. They exist for minutes in duration and are large in magnitude, with many vertical component perturbations in excess of 1000 nT. A possible driving mechanism of these events is transient ionospheric current vorticity associated with the auroral electrojet. Superposed epoch analysis suggests they occur coincidentally with heightened levels of HD in high-latitude sub-auroral substation transformers presenting a potential source of space weather disruption in high latitude sub-auroral and auroral zone ground-based infrastructure. The demonstrated presence and geoeffectiveness of these vertical impulsive events presents a space weather hazard to auroral-zone and high-latitude sub-auroral power transmission networks. This emphasizes the need for improved threedimensional geoelectric field models. The extent of the space weather hazard posed by vertical impulsive geomagnetic events may not be captured by geoelectric field models that neglect the vertical component of the geomagnetic field.

Acknowledgements. For the SML and SMU indices, we gratefully acknowledge the SuperMAG collaborators (https:// supermag.jhuapl.edu/info/?page=acknowledgement). SuperMAG data sets are available from their repository (https://supermag. jhuapl.edu/). AUTUMNX is supported by CSA funding (http:// autumn.athabascau.ca), with open data access to magnetic data used in this study. We thank Ian Schofield for his role in the installation and operation of the AUTUMNX array. Harmonic distortion data was provided to us by Hydro-Québec. Archiving historical GIC and HD data is a time-consuming and costly endeavor for which we would like to thank Hydro-Québec, and it is a practice we would encourage other utilities to consider emulating. The editor thanks two anonymous reviewers for their assistance in evaluating this paper.

\section{Supplementary materials}

Supplementary material is available at https://www.swscjournal.org/10.1051/swsc/2021029/olm

Figure S1. Example of a candidate event (red circle) from February 2, 2017 which meets all selection criteria.

Figure S2. Example of a candidate event (red circle) from February 13, 2019 which does not meet selection criterion 1, $d B / d t<0.5 \mathrm{nT}$. This criterion is used to select for local extrema in the magnetic field component.

Figure S3. Example of a candidate event (red circle) from April 10, 2019 which does not meet the selection criterion for the lowest or highest extrema in a five-minute window.

Figure S4. An example of a candidate event (red circle) from January 6, 2015 which does not meet the selection criterion for a difference between the extremum magnetic component value and median component values in a four minute window on both sides of the extremum.

Figure S5. An example of a candidate event (red circle) February 17, 2016 which does not meet the power spectral density selection criterion. This selects for non-recurrent impulsive magnetic impulses, from data in a two hour window around an extremum.

Figure S6. An example of a candidate event (red circle) from November 8, 2019 which does not meet the selection citerion for median $d B / d t$ in a 10 minute window around the extremum. As seen, this selects for events with significant median $\mathrm{dBdt}$ around the extremum, and often finds instrumentation errors.

Figure S7. An example of a candidate event (red circle) from January 7, 2015 which was discarded in the human investigation step. Candidate event satisfied other selection criteria but is not a significant impulsive event as compared with local magnetic field component fluctuations.

\section{References}

Albertson VD, Kappenman JG, Mohan N, Skarbakka GA. 1981. Load-flow studies in the presence of geomagnetically-induced currents. IEEE Trans Power Appar Syst PAS-100(2): 594-607. https://doi.org/10.1109/TPAS.1981.316916.

Belakhovsky V, Pilipenko V, Engebretson M, Sakharov Y, Selivanov V. 2019. Impulsive disturbances of the geomagnetic field as a cause of induced currents of electric power lines. $J$ Space Weather Space Clim 9: A18. https://doi.org/10.1051/swsc/ 2019015. 
Bolduc L. 2002. GIC observations and studies in the Hydro-Québec power system. J Atmos Sol-Terr Phys 64(16): 1793-1802. https://doi.org/10.1016/S1364-6826(02)00128-1.

Boteler DH. 2001. Assessment of geomagnetic hazard to power systems in Canada. Nat Hazards 23(2-3): 101-120. https://doi. org/10.1023/A:1011194414259.

Boteler DH. 2003. Geomagnetic hazards to conducting networks. Nat Hazards 28(2-3): 537-561. https://doi.org/10.1023/ A:1022902713136.

Boteler DH. 2019. A 21st century view of the March 1989 magnetic storm. Space Weather 17(10): 1427-1441. https://doi.org/10.1029/ 2019 SW002278.

Boteler DH, Pirjola RJ. 2017. Modeling geomagnetically induced currents. Space Weather 15(1): 258-276. https://doi.org/10.1002/ 2016 SW001499.

Chinkin VE, Soloviev AA, Pilipenko VA, Engebretson MJ, Sakharov Y. 2021. Determination of vortex current structure in the high-latitude ionosphere with associated GIC bursts from ground magnetic data. J Atmos Sol-Terr Phys 212 (November 2020): 105,514. https://doi.org/10.1016/j.jastp.2020. 105514.

Clilverd MA, Rodger CJ, Brundell JB, Dalzell M, Martin I, Mac Manus DH, Thomson NR. 2020. Geomagnetically induced currents and harmonic distortion: High time resolution case studies. Space Weather 18(10): e2020SW002594. https://doi.org/ 10.1029/2020SW002594.

Connors M, Schofield I, Reiter K, Chi PJ, Rowe KM, Russell CT. 2016. The AUTUMNX magnetometer meridian chain in Québec, Canada. Earth Planets Space 68(1): 2. https://doi.org/10.1186/ s40623-015-0354-4.

Danskin DW, Lotz SI. 2015. Analysis of geomagnetic hourly ranges. Space Weather 13(8): 458-468. https://doi.org/10.1002/ 2015SW001184.

Engebretson MJ, Yeoman TK, Oksavik K, Søraas F, Sigernes F, et al. 2013. Multi-instrument observations from Svalbard of a traveling convection vortex, electromagnetic ion cyclotron wave burst, and proton precipitation associated with a bow shock instability. J Geophys Res: Space Phys 118(6): 2975-2997. https://doi.org/10.1002/jgra.50291.

Engebretson MJ, Pilipenko VA, Ahmed LY, Posch JL, Steinmetz ES, et al. 2019a. Nighttime magnetic perturbation events observed in Arctic Canada: 1. Survey and statistical analysis. J Geophys Res: Space Phys 124(9): 7442-7458. https://doi.org/10.1029/ 2019JA026794.

Engebretson MJ, Steinmetz ES, Posch JL, Pilipenko VA, Moldwin $\mathrm{MB}$, et al. 2019b. Nighttime magnetic perturbation events observed in Arctic Canada: 2. Multiple-instrument observations. J Geophys Res: Space Phys 124(9): 7459-7476. https://doi.org/ 10.1029/2019JA026797.

Frey HU, Mende SB, Angelopoulos V, Donovan EF. 2004. Substorm onset observations by IMAGE-FUV. J Geophys Res: Space Phys 109(A10): 1-6. https://doi.org/10.1029/2004JA010607.

Friis-Christensen E, McHenry MA, Clauer CR, Vennerstrøm S. 1988. Ionospheric traveling convection vortices observed near the polar cleft: A triggered response to sudden changes in the solar wind. Geophys Res Lett 15(3): 253-256. https://doi.org/10.1029/ GL015i003p00253.

Fukushima N. 1994. Some topics and historical episodes in geomagnetism and aeronomy. J Geophys Res 99(A10): 19,113. https://doi.org/10.1029/94ja00102.

Gjerloev JW. 2012. The SuperMAG data processing technique. J Geophys Res: Space Phys 117(A9): A09213. https://doi.org/ 10.1029/2012JA017683.
Guillon S, Toner P, Gibson L, Boteler D. 2016. A colorful blackout. IEEE Power Energy Mag 14(November/December): 59-71. https://doi.org/10.1109/MPE.2016.2591760.

Harang L. 1946. The mean field of disturbance of polar geomagnetic storms. Terr Magn Atmos Elect 51(3): 353-380. https://doi.org/ 10.1029/TE051i003p00353.

Harris SE, Mende SB, Angelopoulos V, Rachelson W, Donovan E, et al. 2009. THEMIS ground based observatory system design, Springer New York, New York, NY, pp. 213-233. ISBN 978-0387-89820-9. https://doi.org/10.1007/978-0-387-89820-9_10.

Hruska J, Coles RL. 1987. A new type of magnetic activity forecast for high geomagnetic latitudes. J Geomagn Geoelect 39(9): 521534. https://doi.org/10.5636/jgg.39.521.

Kamide Y. 1978. On current continuity at the Harang discontinuity. Planet Space Sci 26(3): 237-244. https://doi.org/10.1016/00320633(78)90089-2.

Kamide Y, Vickrey JF. 1983. Variability of the Harang discontinuity as observed by the Chatanika radar and the IMS Alaska magnetometer chain. Geophys Res Lett 10(2): 159-162. https://doi.org/10.1029/GL010i002p00159.

Kamwa I, Béland J, Trudel G, Grondin R, Lafond C, McNabb D. 2006. Wide-area monitoring and control at Hydro-Québec: Past, present and future. In: 2006 IEEE Power Engineering Society General Meeting, PES, Vol. 7. IEEE, Montréal, QC, Canada, p. 12. https://doi.org/10.1109/pes.2006.1709097.

Kanasewich ER. 1974. Time sequence analysis in geophysics, Canadian Electronic Library. Canadian publishers collection. University of Alberta Press, Edmonton. ISBN 1-4593-0464-0.

Kappenman JG. 2005. An overview of the impulsive geomagnetic field disturbances and power grid impacts associated with the violent Sun-Earth connection events of 29-31 October 2003 and a comparative evaluation with other contemporary storms. Space Weather 3(8): S08C01. https://doi.org/10.1029/2004SW000128.

Kisabeth JL, Rostoker G. 1974. The expansive phase of magnetospheric substorms: 1. Development of the auroral electrojets and auroral arc configuration during a substorm. J Geophys Res 79(7): 972-984. https://doi.org/10.1029/ja079i007p00972.

Lanzerotti LJ, Lee LC, Maclennan CG, Wolfe A, Medford LV. 1986. Possible evidence of flux transfer events in the polar ionosphere. Geophys Res Lett 13(11): 1089-1092. https://doi.org/10.1029/ GL013i011p01089.

Lanzerotti LJ, Konik RM, Wolfe A, Venkatesan D, Maclennan CG. 1991. Cusp latitude magnetic impulse events: 1. Occurrence statistics. J Geophys Res: Space Phys 96(A8): 14,009-14,022. https://doi.org/10.1029/91ja00567.

Lundstedt H. 2006. The sun, space weather and GIC effects in Sweden. Adv Space Res 37(6): 1182-1191. https://doi.org/ 10.1016/j.asr.2005.10.023.

McPherron RL, Russell CT, Aubry MP. 1973. Satellite studies of magnetospheric substorms on August 15, 1968: 9. Phenomenological model for substorms. J Geophys Res 78(16): 3131-3149. https://doi.org/10.1029/ja078i016p03131.

Moretto T, Sibeck DG, Watermann JF. 2004. Occurrence statistics of magnetic impulsive events. Ann Geophys 22(2): 585-602. https://doi.org/10.5194/angeo-22-585-2004.

Newell PT, Gjerloev JW. 2011. Evaluation of SuperMAG auroral electrojet indices as indicators of substorms and auroral power. J Geophys Res: Space Phys 116(A12): A12211. https://doi.org/ 10.1029/2011JA016779.

Ngwira CM, Pulkkinen AA, Bernabeu E, Eichner J, Viljanen A, Crowley G. 2015. Characteristics of extreme geoelectric fields and their possible causes: Localized peak enhancements. Geophys Res Lett 42(17): 6916-6921. https://doi.org/10.1002/2015GL065061. 
Pulkkinen A, Thomson A, Clarke E, McKay A. 2003. April 2000 geomagnetic storm: Ionospheric drivers of large geomagnetically induced currents. Ann Geophys 21(3): 709-717. https://doi.org/ 10.5194/angeo-21-709-2003.

Rodger CJ, Clilverd MA, Mac Manus DH, Martin I, Dalzell M, et al. et al. 2020. Geomagnetically induced currents and harmonic distortion: Storm-time observations from New Zealand. Space Weather 18(3): e2019SW002,387. https://doi.org/10.1029/2019SW002387.

Stauning P. 2013. Power grid disturbances and polar cap index during geomagnetic storms. J Space Weather Space Clim 3: A22. https://doi.org/10.1051/swsc/2013044.
Untiedt J, Baumjohann W. 1993. Studies of polar current systems using the IMS Scandinavian magnetometer array. Space Sci Rev 63(3-4): 245-390. https://doi.org/10.1007/BF00750770.

Wait JR. 1982. Geo-electromagnetism, Academic Press, New York, NY. ISBN 0127308806. https://doi.org/10.1016/B978-0-12-7308807.X5001-7.

Zou S, Lyons LR, Nicolls MJ, Heinselman CJ, Mende SB. 2009. Nightside ionospheric electrodynamics associated with substorms: PFISR and THEMIS ASI observations. J Geophys Res: Space Phys 114(12): 1-24. https://doi.org/10.1029/2009JA014259.

Cite this article as: Reiter K, Guillon S, Connors M \& Jackel B 2021. Statistics of large impulsive magnetic events in the auroral zone. J. Space Weather Space Clim. 11, 44. https://doi.org/10.1051/swsc/2021029. 